\title{
An unusual epithelial pleomorphic giant cell tumour of the pancreas with osteoclast-type cells
}

\author{
A Martin, P Texier, J M Bahnini, J Diebold
}

\begin{abstract}
A case of giant cell tumour of the pancreas with a mixture of pleomorphic giant cells and osteoclast-like cells is described. This association is rare and its histogenesis has been debated. The presence of a small differentiated adenocarcinomatous area at the periphery of the tumour indicates an epithelial origin. Moreover, some pleomorphic cells were positive for keratin (KL1). The osteoclast-like cells strongly expressed CD68 (a marker of histiomonocytic lineage) and did not show proliferative activity. They probably correspond to an unusual reaction of the stroma. Their clinical importance in this type of tumour remains unknown.
\end{abstract}

( Clin Pathol 1994;47:372-374)

Of the non-endocrine tumours of the pancreas, giant cell tumours are rare. Two histopathological types can usually be distinguished. ${ }^{12}$ The former is described as a pleomorphic giant cell carcinoma. It generally corresponds to an undifferentiated sarcomalike tumour, composed of atypical mononuclear and multinucleated giant cells. Its unfavourable prognosis is similar to that of pancreatic adenocarcinomas. ${ }^{1}$ The second type corresponds to a giant osteoclast-like cell tumour or osteoclastoma, the histopathological pattern of which is similar to skeletal giant cell tumours. ${ }^{3-6}$ Its prognosis is usually better than that of pleomorphic giant cell carcinoma. 46

The histogeneses of these tumours have been discussed. The epithelial nature of the pleomorphic giant cell tumour is well known. ${ }^{1}$ For giant osteoclast-like cell tumours, several studies have reported features of epithelial differentiation ${ }^{347}$; others have referred to a mesenchymal nature. ${ }^{23}$ The distinction between these two types of giant cell tumour is not always clear. For example, some association between pleomorphic giant cell tumours and osteoclast-like cell tumours has been described, indicating a possible overlap between the two types. ${ }^{1278}$

\section{Case report}

A 57 year old man was admitted to hospital in February 1992 following left flank pain with irradiated back pain. There was no prior notable loss of weight or gastrointestinal symptoms. No lymphadenopathy or hepatosplenomegaly were discovered by physical examination.

An abdominal computed tomogram demonstrated a splenic mass in the tail of the pancreas and lateral aortic lymphadenopathies. A large tumour was found on laparotomy, which expanded into the hilum of the spleen, the transverse mesocolon, and the posterior wall of the stomach. No hepatic metastasis was discerned. A distal pancreatectomy, with splenectomy and total gastrectomy "en bloc", was performed.

After a short respite the patient rapidly deteriorated. He died four months later, after a second laparotomy which indicated a peritoneal carcinomatosis. Necropsy was not carried out.

\section{Methods}

After fixation in $10 \%$ formalin, multiple blocks taken from the tumour were embedded in paraffin wax. For light microscopy, $5 \mu \mathrm{m}$ sections were stained with haematoxylin and eosin and safran, periodic acid Schiff with diastase digestion, and alcian blue.

An immunohistochemical study using the streptavidin-biotin peroxidase method (Amersham International, Buckinghamshire, England), revealed by 3-amino-9-ethyl-carbazol (Sigma, St Louis, USA), was performed on $5 \mu \mathrm{m}$ representative paraffin wax embedded sections of the tumour. Antibodies to the following antigens were tested: cytokeratin KL1, epithelial membrane antigen (EMA), vimentin, desmin, actin (Immunotech, Marseille, France), carcino-embryonic antigen (CEA) (Cambridge Research Laboratories), 40-52 kilodaltons keratin, 52-58 kilodaltons keratin (Eurodiagnostic, Ajeldooin, The Netherlands), CD45, lysozyme, $a-1$ antitrypsin, CD68 (KP1), and proliferation cell nuclear antigen (PCNA) PC10 (Dakopatts, Glostrup, Denmark).

\section{Pathological findings}

The surgical resection specimen contained a $7 \times 5,5 \times 5 \mathrm{~cm}$ tumour in the tail of the pancreas. Sections showed a proliferation of non-cohesive pleomorphic cells with numerous giant cells, and exhibited an abundant cytoplasm, occasionally vacuolated, and sometimes faintly stained by alcian blue. They contained an irregular and often polylobulated nucleus with one or several large nucleoli. Numerous mitoses were present.
Correspondence to: Dr A Martin

Accepted for publication 21 September 1993
Service Central d'Anatomi Dieu, 1 place du A Martin

Clinique Pasteur, Ris-Orangis, France 
Mixture of pleomorphic and osteoclast-like giant cells. Pleomorphic cells are mixed in with a reactive population of small cells. Osteoclast-like cells are composed of a large, darkly stained cytoplasm and numerous small nuclei (haematoxylin and eosin). Inset, immunohistochemical staining. All the osteoclast-like cells intensely expressed CD68 (KP1).

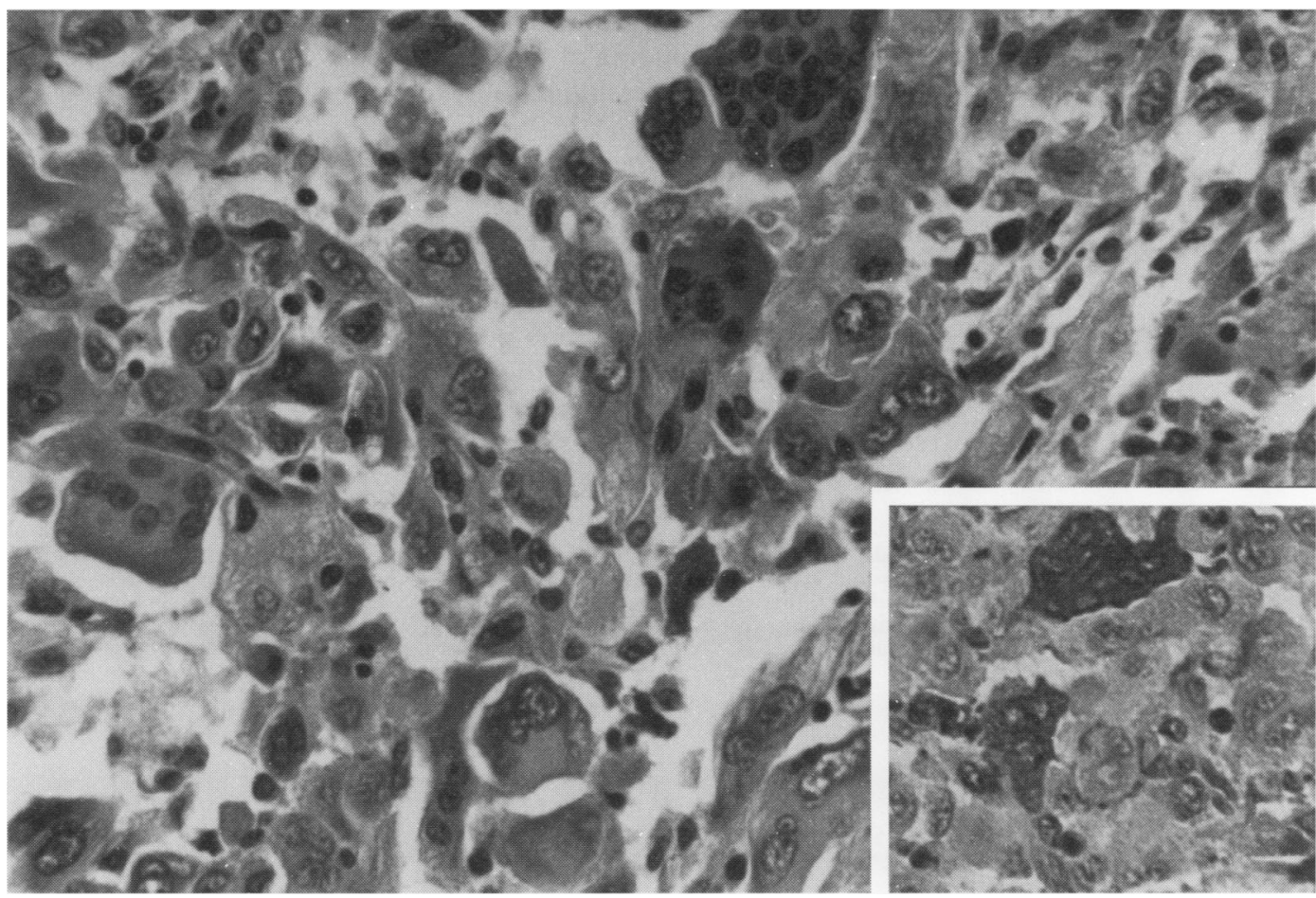

Many multinucleated giant cells which looked like osteoclasts were mixed up with pleomorphic cells (figure). These had a large and intensely stained cytoplasm and contained 10 to 50 small, round, and very regular nuclei, frequently concentrated in a central position.

At the periphery of the lesion, adjacent to the pancreatic parenchyma, and in several pancreatic nodes, differentiated adenocarcinomatous structures were found. There was no mixture of the two tumour patterns.

\section{IMMUNOHISTOCHEMICAL FINDINGS}

The pleomorphic cells were variably stained by vimentin and weakly by keratin $\mathrm{KL} L$. The other epithelial markers were negative (EMA, 40-52 and 52-58 kilodaltons). The osteoclast-like cells were not stained by lysozyme and $a-1$ anti-trypsin antibodies. However, these cells strongly expressed CD68, indicating a histiomonocytic origin (figure). Vimentin was weakly expressed. CD45 did not stain and there was no immunohistochemical feature of epithelial differentiation. The adenocarcinoma structures expressed only the epithelial markers and CEA.

In the pleomorphic cells more than $50 \%$ of nuclei expressed PCNA, representing intensive proliferative activity. In contrast, nuclei were not stained in the osteoclast-like cells.

\section{Discussion}

These findings highlight the difficulty involved in the nosological classification of the giant cell tumours of the pancreas. Indeed, this case like some previous cases ${ }^{128}$ combines the features of a pleomorphic giant cell tumour with an osteoclast-like cell tumour.

This raises the question of the exact origin of the different components of the tumour.
For osteoclast-like cell tumours, Rosaï described some ultrastructural features of epithelial differentiation. ${ }^{4}$ Other authors have also attributed an epithelial origin from ultrastructural findings, ${ }^{8}$ but features mentioned in their studies, such as microvilli and desmosome-like junctions, have also been described in some mesenchymal tumours. ${ }^{7}$ The authors of the present study believe, as do others, ${ }^{1}$ that the osteoclast-like cells might represent a reactive component of the tumoural stroma.

In the present case, histiomonocytic differentiation of the osteoclast-like cells was shown by the strong expression of CD68. Moreover, the benign cytological results and the lack of PCNA expression are additional arguments in support of a reactive nature for these cells.

The epithelial origin of the tumour is attested by the existence of rare adenocarcinomatous structures. The conjunction of two different tumours is improbable. The pleomorphic component would correspond to the emergence of a less differentiated clone, which would have lost most of the epithelial markers and CEA initially present on the differentiated adenocarcinomatous glands.

Mixtures of pleomorphic giant cell tumours with adenocarcinomatous structures do occur. ${ }^{1}$ Some reports have mentioned an association of osteoclast-like cell tumours with adenocarcinoma. ${ }^{179}$

Primary extraskeletal tumours with osteoclast-like giant cells are not specific to the pancreas, and some cases have been described in other organs, such as the breast, thyroid, parotid gland, soft tissue, and colon (for a review see Berendt et $a l^{3}$ ).

Some pancreatic giant cell tumours could have a true mesenchymal origin. Malignant fibrous histiocytomas of the pancreas with giant cells have been described. ${ }^{10}$ It is difficult 
to distinguish a true mesenchymal tumour from a giant cell carcinoma of the pancreas in the absence of adenocarcinomatous components.

The clinical importance of the osteoclastlike cells in pancreatic tumours, as in other locations, remains to be clarified. They may increase the aggressiveness of the tumour by producing an abundant quantity of proteolytic enzymes. Different factors produced by some tumours could stimulate the differentiation of circulating precursor cells into the osteoclast.

1 Cubilla AL, Fitzgerald PJ. Giant cell carcinoma (osteoclastoïd type). In: Firminger HI, ed. Atlas of tumor pathology, Series 2, Fascicle 19. Washington, DC: Armed Forces Institute of Pathology, 1984:162-7.

2 Lewandrowsky KB, Weston L, Dickersin R, Rattner DW,
Compton CC. Giant cell tumor of the pancreas of mixed osteoclastic and pleomorphic cell type: evidence for a histogenetic relationship and mesenchymal differentiation. Hum Pathol 1990;21:1184-7.

3 Berendt RC, Shnitka TK, Wiens E, Manickavel V, Jewell LD. The osteoclast-type giant cell tumor of the pancreas. Arch Pathol Lab Med 1987;111:43-8.

4 Rosai J. Carcinoma of the pancreas simulating giant cell tumor of bone. Electron-microscopic evidence of its tumor of bone. Electron-microscopic evid

5 Jalloh SS. Giant cell tumour (osteoclastoma) of the pancreas: an epithelial tumour probably of pancreatic acinar origin. 7 Clin Pathol 1983;36:1171-5.

6 Jeffrey I, Crow J, Ellis BW. Osteoclast-type giant cell of the pancreas. $\mathscr{f}$ Clin Pathol 1983;36:1 165-70.

7 Trepeta RW, Mathur B, Lagin S, Livolsi VA. Giant cell tumor ("osteoclastoma") of the pancreas. A tumor of epithelial origin. Cancer $1981 ; 48: 2022-8$.

8 Fisher HP, Altmannsberger M, Kracht J. Osteoclast-type giant cell tumor of the pancreas. Virchows Arch (Pathol Anat) 1988;412:247-53.

9 Posen JA, Path FF. Giant cell tumor of the pancreas of the osteoclastic type associated with a mucous secreting cystadenocarcinoma. Hum Pathol 1981;12:944-7.

10 Suster S, Philip M, Robinson MJ. Malignant fibrous histiocytoma (giant cell type) of the pancreas. A distinctive variant of osteoclast-type giant cell tumor of the pancreas. Cancer 1989;64:2303-8. 